Review Article

\title{
Gastrectomy and D2 Lymphadenectomy for Gastric Cancer: A Meta-Analysis Comparing the Harmonic Scalpel to Conventional Techniques
}

\author{
Hang Cheng, ${ }^{1}$ Chia-Wen Hsiao, ${ }^{1}$ Jeffrey W. Clymer, ${ }^{1}$ Michael L. Schwiers, ${ }^{1}$ \\ Bryanna N. Tibensky, ${ }^{2}$ Leena Patel, ${ }^{2}$ Nicole C. Ferko, ${ }^{2}$ and Edward Chekan ${ }^{1}$ \\ ${ }^{1}$ Ethicon Inc., 4545 Creek Road, Cincinnati, OH 45242, USA \\ ${ }^{2}$ Cornerstone Research Group, 204-3228 South Service Road., Burlington, ON, Canada L7N 3H8
}

Correspondence should be addressed to Jeffrey W. Clymer; jclymer@its.jnj.com

Received 18 February 2015; Revised 26 March 2015; Accepted 1 April 2015

Academic Editor: Kazuhiro Yoshida

Copyright (C) 2015 Hang Cheng et al. This is an open access article distributed under the Creative Commons Attribution License, which permits unrestricted use, distribution, and reproduction in any medium, provided the original work is properly cited.

The ultrasonic Harmonic scalpel has demonstrated clinical and surgical benefits in dissection and coagulation. To evaluate its use in gastrectomy, we conducted a systematic review and meta-analysis of randomized controlled trials comparing the Harmonic scalpel to conventional techniques in gastrectomy for patients with gastric cancer. International databases were searched without language restrictions for comparisons in open or laparoscopic gastrectomy and lymphadenectomy. The meta-analysis used a random-effects model for all outcomes; continuous variables were analyzed for mean differences and dichotomous variables were analyzed for risk ratios. Sensitivity analyses were conducted for study quality, type of conventional technique, and imputation of study results. Ten studies $(N=935)$ met the inclusion criteria. Compared with conventional hemostatic techniques, the Harmonic scalpel demonstrated significant reductions in operating time $(-27.5 \mathrm{~min} ; P<0.001)$, intraoperative blood loss $(-93.2 \mathrm{~mL} ; P<0.001)$, and drainage volume $(-138.8 \mathrm{~mL} ; P<0.001)$. Results were numerically higher for conventional techniques for hospital length of stay, complication risk, and transfusions but did not reach statistical significance. Results remained robust to sensitivity analyses. This meta-analysis demonstrates the clear advantages of using the Harmonic scalpel compared to conventional techniques, with improvements demonstrated across several outcome measures for patients undergoing gastrectomy and lymphadenectomy.

\section{Introduction}

Gastric cancer (GC) is the fourth most frequently occurring cancer in the world, with 989,600 new cases ( $8 \%$ of total) and 738,000 deaths ( $10 \%$ of total) estimated worldwide in 2008 $[1,2]$. GC has a significant global burden, with a particularly high incidence in Eastern Europe, South America, and Eastern Asia [2,3]. Several factors are associated with an increased risk for GC, the most common including Helicobacter pylori infection and smoking $[4,5]$. Until recently, the most common forms of GC were fundus and distal gastric cancers; however, there has recently been a shift to a greater prevalence of adenocarcinoma [3]. Current treatment guidelines recommend the use of surgical gastric resection for the management of resectable GC; however, there is variation between countries regarding the extent of lymphadenectomy that is performed alongside gastrectomy $[1,3,6,7]$.
Traditionally, hemostasis during gastrectomy has been achieved using a variety of techniques, including suture ligation and monopolar electrosurgery. However, the use of these conventional techniques has been associated with several challenges. For instance, suture ligation not only is a timeconsuming process, but is also associated with the risk of knot slipping [8]. Further, in monopolar electrosurgery, the high temperatures $\left(150^{\circ} \mathrm{C}-400^{\circ} \mathrm{C}\right)$ that result from using electrical energy to cauterize the tissue can spread into neighboring structures, increasing the risk of injuring surrounding tissue $[8,9]$.

Ultrasonic devices have been developed to address the challenges associated with conventional hemostasis techniques. Through its design, the Harmonic scalpel simultaneously cuts and coagulates tissue. The device converts electrical energy into mechanical energy, causing the blade to vibrate 
at a frequency of $55.5 \mathrm{kHz}$ [9]. Simultaneous cutting and coagulation of tissue are achieved by the high frequency vibration of the blade, which generates stress and friction in the tissue molecules. In turn, this disrupts hydrogen bonds and generates heat, causing protein molecules in the tissue to denature and adhere to one another, thereby forming a hemostatic seal. Importantly, no electrical current is passed through the patient, which reduces the risk of burns and injury. Additionally, unlike monopolar electrosurgery, the Harmonic scalpel operates at lower temperatures, thereby dispersing less energy to surrounding tissues and reducing the risk of thermal damage $[9,10]$.

Several studies have demonstrated the clinical and surgical advantage of the Harmonic scalpel over conventional techniques in different surgical areas, including thyroidectomy [10-13], cholecystectomy $[14,15]$, and colectomy [1619]. Furthermore, this device has been widely used in both laparoscopic and open surgeries [10]. More recently, a number of randomized controlled trials (RCTs) have investigated the use of this device compared to conventional techniques in gastrectomy $[20,21]$. In this procedure, the Harmonic device is typically used for tissue dissection and sealing of smaller to moderately sized blood and lymphatic vessels, while hemoclips or surgical ties are used for major blood vessels, such as the gastric or gastroepiploic vessels. Results from these studies demonstrate that the Harmonic scalpel can significantly improve outcomes such as operating time in gastrectomy procedures. Further, comparison of ultrasonic devices to conventional methods in a meta-analysis of RCTs and observational studies performed by Chen and colleagues [22] associated beneficial outcomes with ultrasonic scalpels in open gastrectomy procedures.

Presently, there are no meta-analyses examining the exclusive impact of the Harmonic scalpel in gastrectomy. Consequently, this systematic review and meta-analysis paper was conducted to evaluate the performance of the Harmonic scalpel versus conventional techniques in both open and laparoscopic gastric surgery for patients with gastric cancer.

\section{Methods}

A systematic search of 21 databases was conducted, including MEDLINE via PubMed, the Cochrane Central Register of Controlled Trials (CENTRAL), EMBASE, and 18 other national databases (Table 1). Comprehensive searches were additionally conducted using Google Scholar and ResearchGate. Reference lists of retrieved articles were hand-searched. No language restrictions were applied.

Specific inclusion criteria were defined according to PICOS (i.e., population, intervention, comparator, outcomes, and study design). Studies were considered eligible for inclusion if they were RCTs comparing the use of Harmonic surgical devices to conventional methods, such as monopolar electrosurgery and suture, clips, or knot-tying (Table 2), in human subjects, for all types of surgery. Full-text studies were then excluded firstly if they did not focus on either open or laparoscopic gastrectomy for gastric cancer, secondly
TABLE 1: List of databases and search periods included in systematic search.

\begin{tabular}{lc}
\hline Databases & Search dates \\
\hline EMBASE & Until 30 September \\
MEDLINE (via PubMed) & 2013 \\
CENTRAL & \\
\hline
\end{tabular}

LILACS and IBECS

African Index Medicus, Index Medicus for

Eastern and Mediterranean

Region, and Index Medicus for South-East Asia

Region and The Western Pacific

Region Index Medicus

African Journals Online

IndMed (India)

PakMediNet (Pakistan)

Türk Tip Veri Tabani (Turkey)

Krack (Croatia)

SID and IrMedex (Iran)

KoreaMed (Korea)

\begin{tabular}{ll}
\hline ICHUSHI-Web (Japan) & Until 22 April 2013 \\
\hline
\end{tabular}

Wanfang, Cqvip, and CNKI (China) Until 16 April 2013

if they used advanced energy devices other than Harmonic devices, and thirdly if they used the Harmonic device outside of the cleared indication. In the Harmonic studies that were included, the Methods section had to identify that the Harmonic device was the principal device used for the procedures. Records were evaluated for eligibility by two independent reviewers. Disagreements between reviewers regarding study inclusion were resolved through consensus or by consultation with a third reviewer.

For included studies, details (i.e., baseline characteristics and outcomes) were extracted using a comprehensive data extraction form. Data extraction was conducted by two independent reviewers with discrepancies resolved by consensus or a third party. The data from non-English articles were translated, extracted, and included in the form. One reviewer conducted the data extraction, which was cross-checked by a second reviewer.

Clinical outcome measures included the following: (1) operating time, (2) intraoperative blood loss, (3) drainage volume, (4) length of hospitalization, (5) transfusion risk, and (6) complication rate. When necessary, study authors were contacted for additional methodological details regarding whether open or laparoscopic surgery was performed; however, study authors were not contacted for missing data. Missing variance data were calculated from other effect estimates and dispersion measures where feasible and appropriate. Variance measures (i.e., standard deviation (SD)) were not reported in one study [23] for operating time or in another study [24] for drainage volume. Therefore, the missing variance measures were imputed according to the standard methods outlined by Cochrane [25]. Another study reported all continuous outcomes as medians with $95 \%$ confidence intervals (CI) [26]; therefore, assuming a normal distribution, the data were imputed using methods outlined by Cochrane [25] and Hozo et al. [27]. 


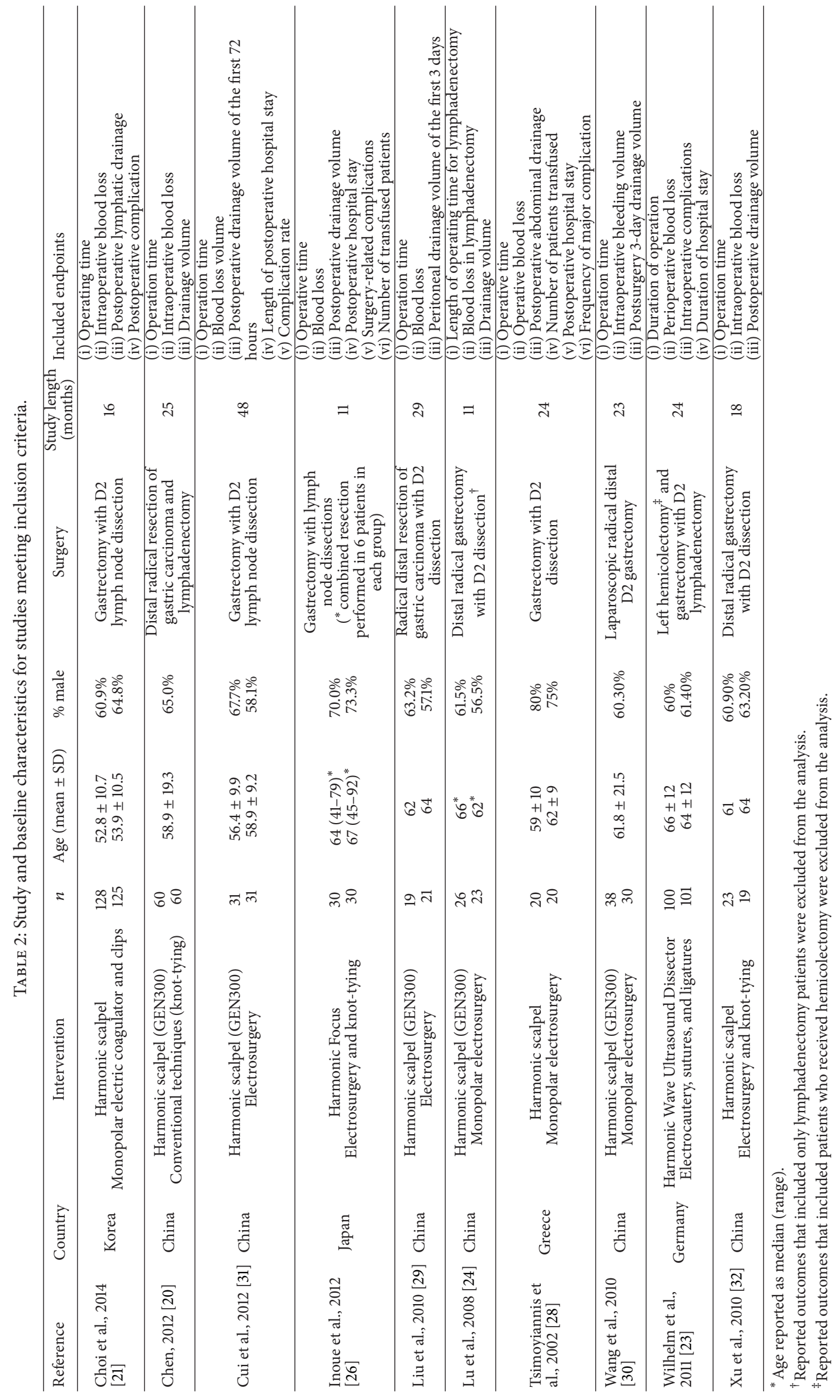




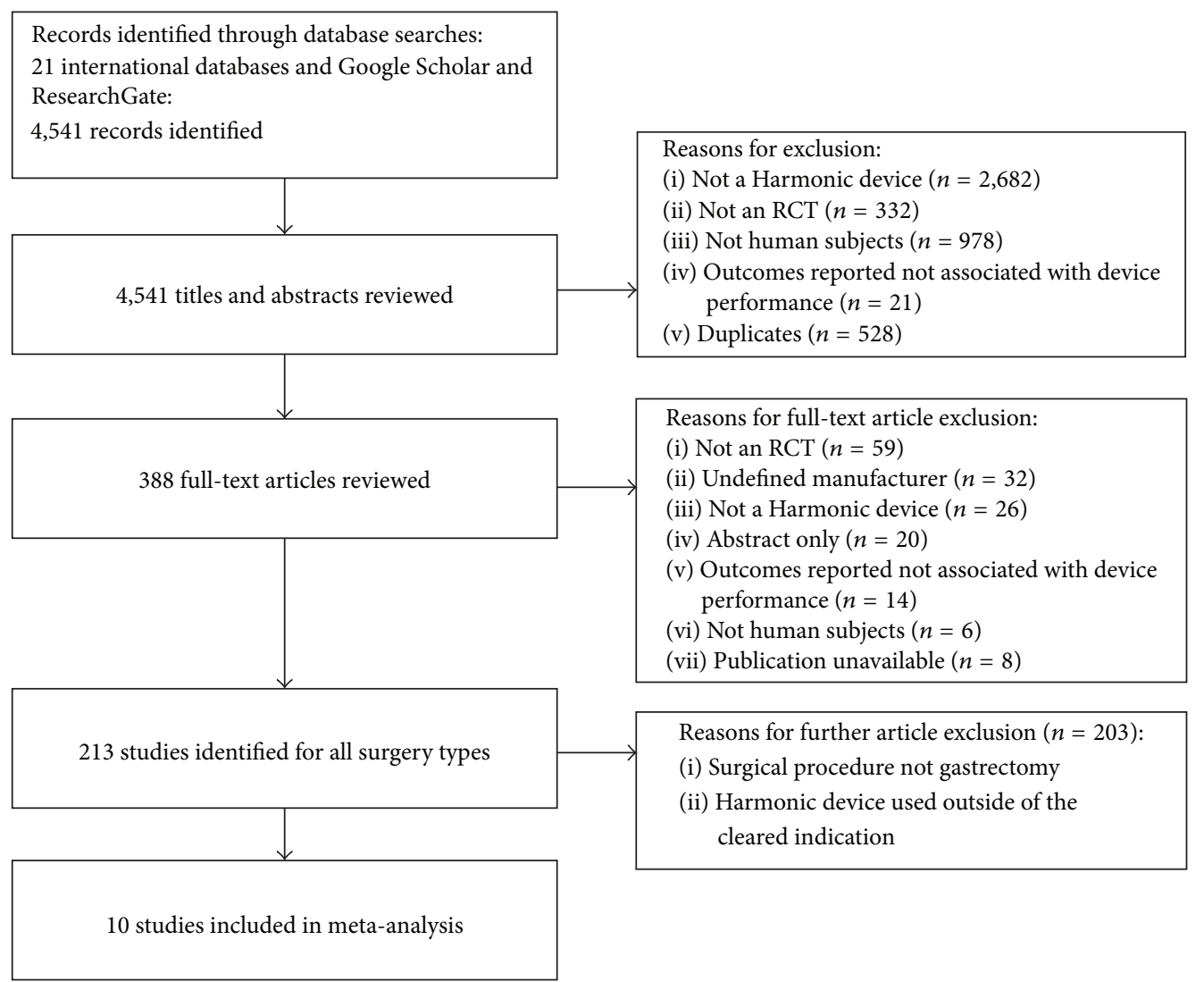

FIGURE 1: PRISMA diagram for the systematic literature review.

The quality of the included studies was assessed using the risk of bias algorithm outlined by the Cochrane guidelines [25]. Studies were scored as having low, unclear, or high risk of bias for the seven domains (sequence generation, allocation concealment, blinding of participants and personnel, blinding of outcome assessment, incomplete outcome data, selective outcome reporting, and other issues) in the assessment tool. Final decisions were dependent on the combination of these factors and the individual characteristics of each study. Study quality was assessed by two independent authors, where disagreements were resolved through consensus or by consultation with a third author.

The meta-analysis was performed using Review Manager (Version 5.3, The Nordic Cochrane Centre, The Cochrane Collaboration, Copenhagen, Denmark, 2014). Continuous variables (operating time, intraoperative blood loss, drainage volume, and length of hospitalization) were analyzed for mean differences (MD) using the inverse-variance method. Dichotomous variables (transfusion risk and complication rate) were analyzed for risk ratios (RR) using the MantelHaenszel method. A random-effects model was used for the meta-analysis and forest plots were generated for all outcomes within Review Manager. Heterogeneity of the included studies was assessed using the $\chi^{2}$ test and $I^{2}$ measure.

The primary analysis compared the Harmonic scalpel to conventional techniques. Sensitivity analyses were completed for study quality, where studies with unclear or high risk of bias across several measures were excluded [20, 26, 28], and for the type of conventional technique used, where studies using only monopolar electrosurgery (i.e., excluding suture ligation) were included [24, 29-31]. Further, sensitivity analyses were conducted by excluding any study outcomes for which imputed data were required.

\section{Results}

A total of 4,541 records were identified from database searching, of which 4,153 were excluded during the title and abstract review (Figure 1). Of the 388 full-text articles retrieved for review, 378 were further excluded if studies were non-RCTs, had an undefined manufacturer, and did not use a Harmonic device within the cleared indication, the publication was unavailable and had nonhuman subjects, or the surgical procedure was not gastrectomy. Ten studies, consisting of a total of 935 patients that reported on the use of Harmonic devices in gastrectomy, were included in the meta-analysis $[20,21,23,24,26,28-32]$.

Study characteristics are presented in Table 2. Sample sizes of the included studies ranged from 40 to 253 patients and study length ranged from 11 to 48 months. In all studies, Harmonic surgical devices (i.e., Harmonic scalpel, Harmonic Wave, and Harmonic Focus) were compared to conventional techniques in gastrectomy. In five studies, the conventional 
TABLE 3: Qualitative risk of bias assessment summary.

\begin{tabular}{|c|c|c|c|c|c|c|c|}
\hline Study & $\begin{array}{l}\text { Sequence } \\
\text { generation }\end{array}$ & $\begin{array}{c}\text { Allocation } \\
\text { concealment }\end{array}$ & $\begin{array}{c}\text { Blinding of } \\
\text { personnel and } \\
\text { participants }\end{array}$ & $\begin{array}{c}\text { Blinding of } \\
\text { outcomes }\end{array}$ & $\begin{array}{c}\text { Incomplete } \\
\text { outcome data } \\
\text { addressed }\end{array}$ & $\begin{array}{l}\text { Free of selective } \\
\text { reporting }\end{array}$ & $\begin{array}{c}\text { Free of other } \\
\text { biases }\end{array}$ \\
\hline Choi et al., 2014 [21] & Yes & Unclear & Yes & Yes & Yes & Yes & Yes \\
\hline Chen, 2012 [20] & No & Unclear & Yes & Yes & Yes & Unclear & Yes \\
\hline Cui et al., 2012 [31] & Yes & Unclear & Unclear & Unclear & Yes & Yes & Yes \\
\hline Inoue et al., 2012 [26] & Unclear & Unclear & Unclear & Unclear & Yes & Yes & Unclear \\
\hline Liu et al., 2010 [29] & Yes & Unclear & Yes & Yes & Unclear & Yes & Yes \\
\hline Lu et al., 2008 [24] & Unclear & Unclear & Yes & Yes & Unclear & Yes & Yes \\
\hline $\begin{array}{l}\text { Tsimoyiannis et al., } 2002 \\
\text { [28] }\end{array}$ & Unclear & Unclear & Unclear & Unclear & Yes & Yes & Yes \\
\hline Wang et al., 2010 [30] & Unclear & Unclear & Yes & Yes & Unclear & Yes & Yes \\
\hline Wilhelm et al., 2011 [23] & Yes & Yes & Yes & Yes & Yes & Yes & Yes \\
\hline Xu et al., 2010 [32] & Unclear & Unclear & Yes & Yes & Yes & Yes & Yes \\
\hline
\end{tabular}

Yes: low risk of bias; No: high risk of bias.

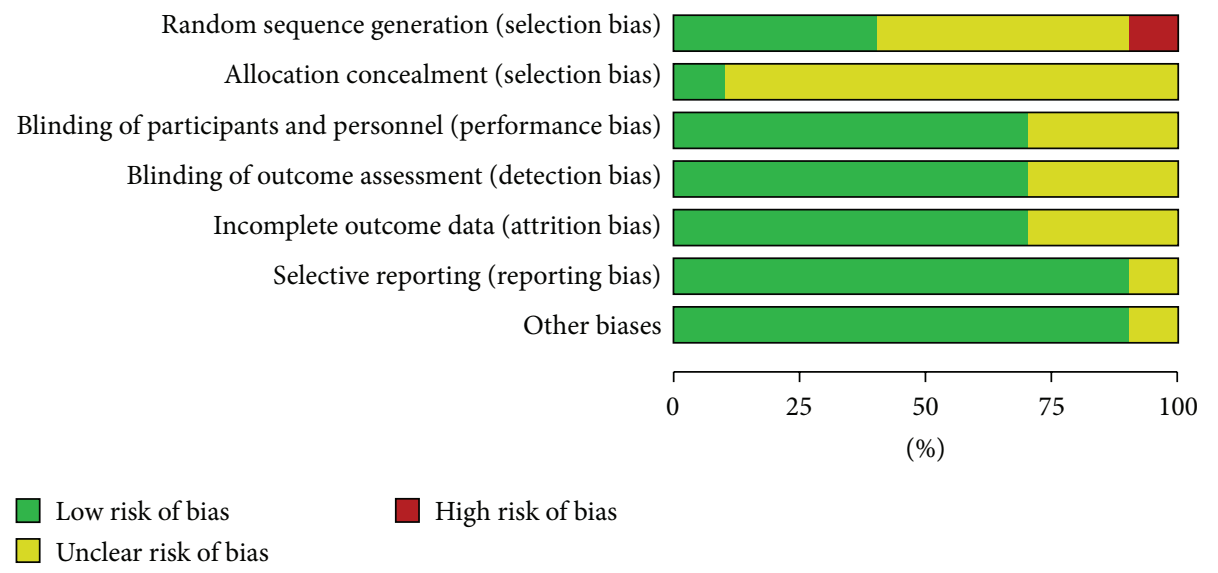

FIGURE 2: Risk of bias assessment for studies meeting inclusion criteria.

method used was monopolar electrosurgery alone [24, 2831]; four studies reported using monopolar electrosurgery in combination with suture ligation or clips $[21,23,26$, 32]; one study did not report the specific conventional methods used [20]. All studies performed lymphadenectomy alongside gastrectomy. Of the ten included studies, five were Chinese; however, patient characteristics were comparable across all included studies. Operating time was reported in nine studies $[20,21,23,26,28-32]$ and eight studies reported intraoperative blood loss [20, 21, 26, 28-32]. One study [24] reported operating time for lymphadenectomy patients only and was therefore excluded from this outcome. Nine studies $[20,21,24,26,28-32]$ reported on drainage volume and three studies reported the postoperative length of hospital stay $[26,28,31]$. Five included studies described the complication rate for both treatment groups $[21,24,26,28,31]$ and the rate for blood transfusions was reported in two studies $[26,28]$. Of the 10 included studies, nine reported on open gastrectomy and one on laparoscopic gastrectomy.

Overall, the risk of bias varied across studies. The overall results of the risk of bias assessments are presented in Figure 2 and the results of the individual study quality assessments are summarized in Table 3. Randomization method was reported in four studies: two studies used a random permuted block design [21, 23], one reported the use of a random number table [31], and one described the use of a lottery system [29]. Only one study [23] described concealment of the randomization sequence. One study [23] reported blinding of patients to the surgical technique. Of the nine studies that did not report blinding, risk of performance bias was deemed low in six studies $[20,21,24,29,30,32]$, where nonblinding was assumed to have no impact on the outcomes assessed. Nine studies reported all prespecified study outcomes; however, selective reporting remained unclear in one study [20]. Attritions or exclusions were reported in two studies [21, 23] but were assumed to have no clinical impact on the observed effect size. In three studies, reporting of attritions or exclusions was insufficient $[24,29,30]$. There were no patient withdrawals in five studies [20,26, 28, 31, 32]. Additional bias was unclear in one study, as there was a reported difference in surgeon status between treatment groups (Harmonic group: $40 \%$ residents versus conventional group: $56.7 \%$ residents) [26]. Studies generally reported positive results which could 


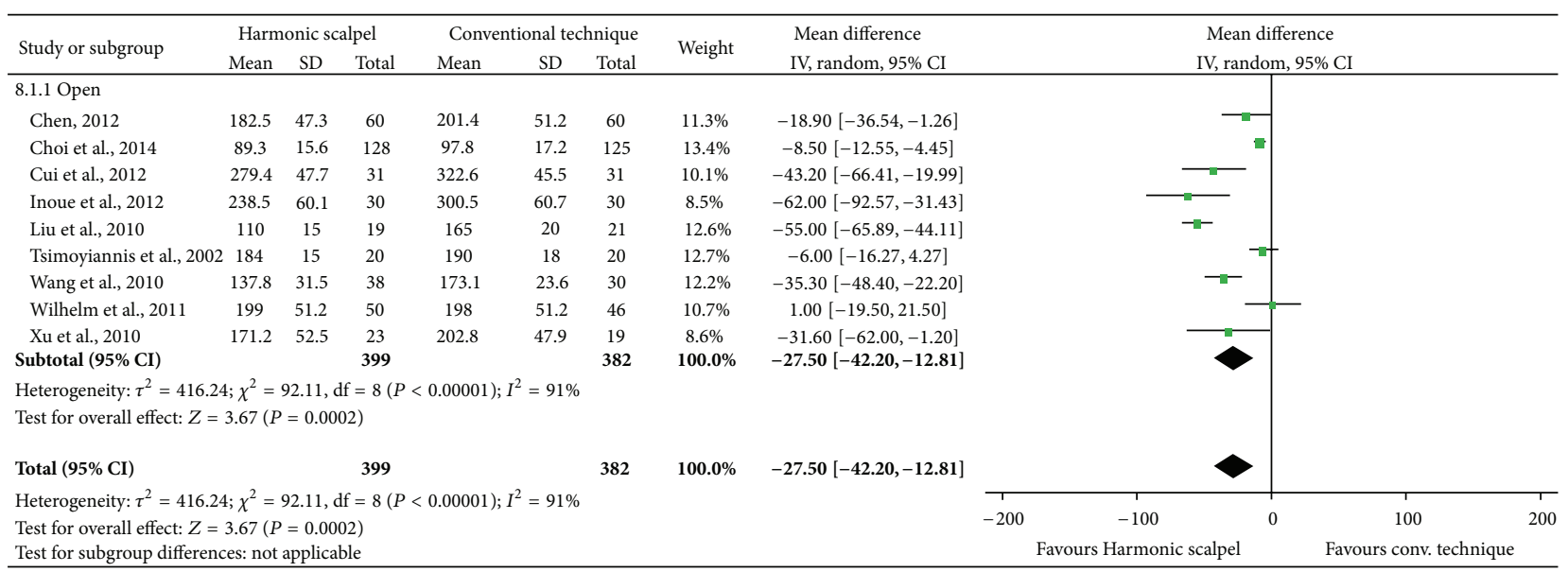

FIGURE 3: Forest plot of meta-analysis results for operating time (minutes), stratified by open versus laparoscopic surgery.

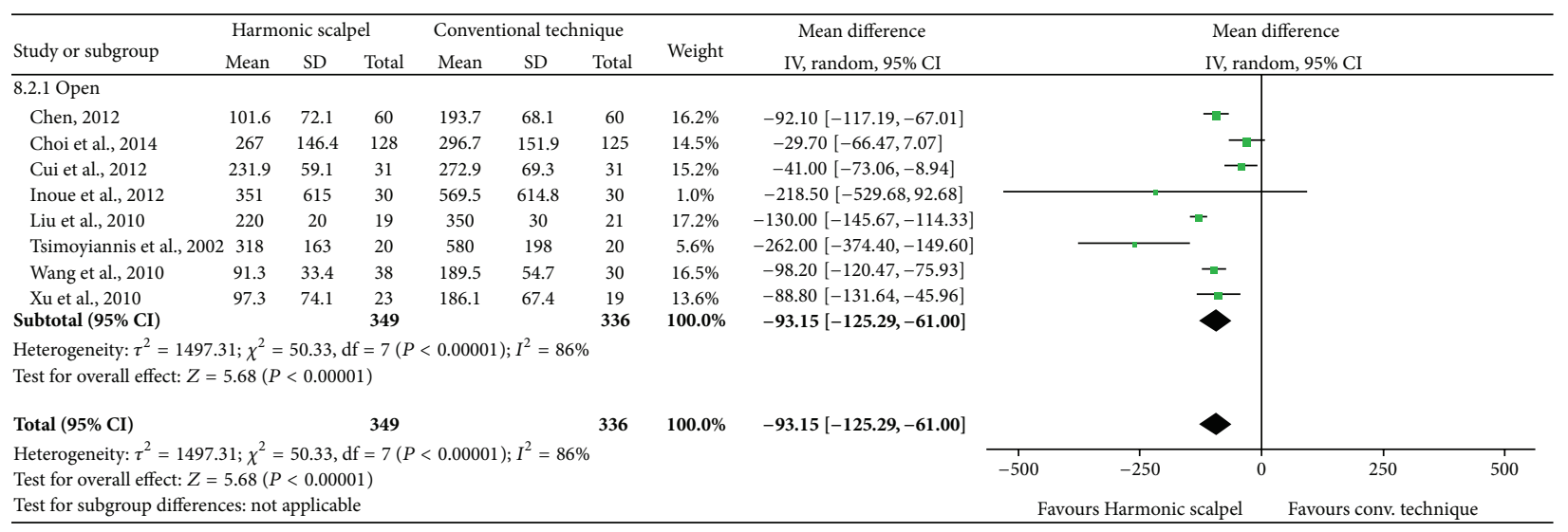

FIGURE 4: Forest plot of meta-analysis results for intraoperative blood loss $(\mathrm{mL})$, stratified by open versus laparoscopic surgery.

result from a publication selection bias; however, funnel plots did not indicate any clear omission of negative studies.

3.1. Operating Time. Mean operating time was statistically significantly reduced by 27.50 minutes (95\% CI: -42.20 to $-12.81 ; P=0.0002 ; 9$ studies; $\left.I^{2}=91 \%\right)$ with the Harmonic scalpel in contrast to conventional methods in open gastrectomy (Figure 3). All results were reported for open gastrectomy.

3.2. Intraoperative Blood Loss. Results demonstrated that, with the Harmonic scalpel, mean intraoperative blood loss was statistically significantly reduced by $93.15 \mathrm{~mL}$ (95\% CI: -125.29 to $-61.00 ; P<0.00001 ; 8$ studies; $\left.I^{2}=86 \%\right)$ in open gastric resection (Figure 4). All results were reported for open gastrectomy.

3.3. Drainage Volume. Compared to conventional techniques, mean drainage volume was statistically significantly reduced $(\mathrm{MD}=-138.83 \mathrm{~mL}$; $95 \% \mathrm{CI}$ : -177.57 to -100.10 ; $P<0.00001 ; 9$ studies; $\left.I^{2}=94 \%\right)$ with the Harmonic scalpel (Figure 5). The study device also showed significant reductions in drainage volume in open surgery $(\mathrm{MD}=$ $-134.36 \mathrm{~mL}$; 95\% CI: -172.86 to $-95.87 ; P<0.00001 ; 8$ studies; $\left.I^{2}=94 \%\right)$. While a subgroup analysis for studies using laparoscopic surgery was not performed due to limited data, the results from the primary analysis and open surgery subgroup analysis were in line with those from the single study that conducted gastrectomy laparoscopically [24].

3.4. Length of Hospital Stay. On the basis of the three studies comparing the Harmonic scalpel to conventional techniques in open gastrectomy, no statistically significant differences in the postoperative length of hospitalization were observed $(\mathrm{MD}=-0.63$ days; $95 \% \mathrm{CI}:-2.48$ to $1.23 ; P=0.51 ; 3$ studies; $I^{2}=65 \%$ ) (Figure 6). All results were reported for open gastrectomy.

3.5. Blood Transfusion. Results demonstrated a lower risk of blood transfusions with the Harmonic scalpel than with conventional methods, although not statistically significant (RR of 0.68 ; 95\% CI: 0.38 to $1.19 ; P=0.18 ; 2$ studies; $I^{2}=0 \%$ ) (Figure 7). All results were reported for open gastrectomy. 


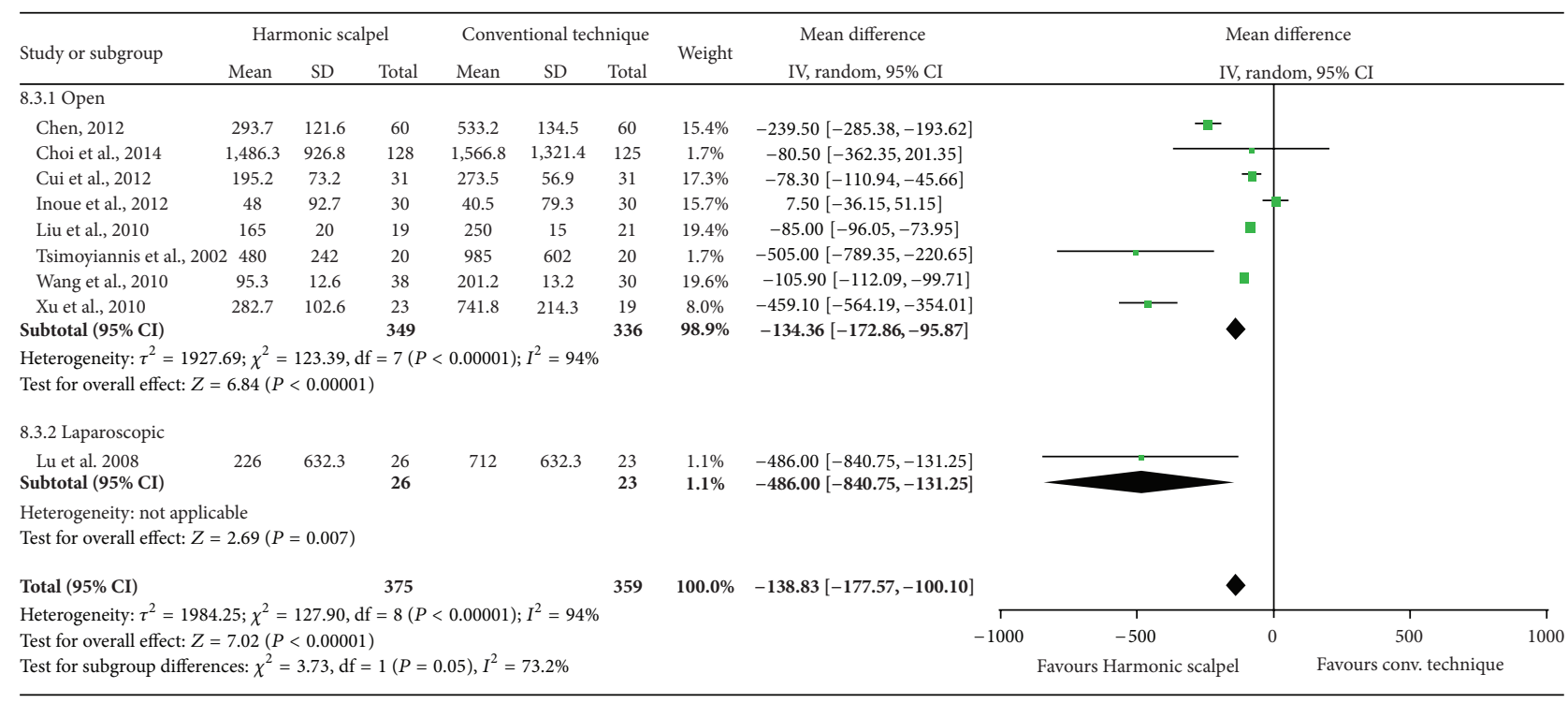

FIGURE 5: Forest plot of meta-analysis results for drainage volume (mL), stratified by open versus laparoscopic surgery.

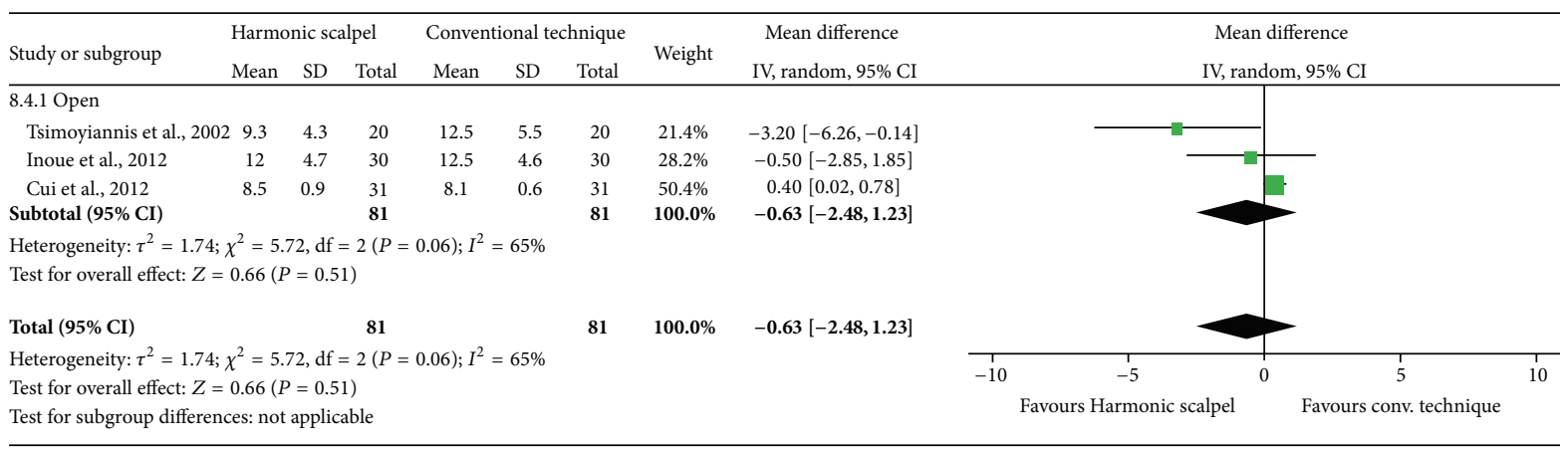

FIGURE 6: Forest plot of meta-analysis results for length of hospital stay (days).

3.6. Postoperative Complications. The main reported complications for both study groups included postoperative bleeding, chylous leakage, gastrointestinal paralysis, and wound infection. The Harmonic scalpel reduced the risk of postoperative complications compared to conventional techniques, although not significantly so $(\mathrm{RR}=0.58$; $95 \% \mathrm{CI}$ : 0.33 to 1.02 ; $P=0.06 ; 5$ studies; $I^{2}=12 \%$ ) (Figure 8 ). Similar results were observed between the open surgery subgroup and the single study reporting on laparoscopic gastric resection.

Sensitivity Analyses. Results of sensitivity analyses on study quality were similar to the primary analysis and were relatively robust to variables tested with some exceptions. For operating time, intraoperative blood loss, and drainage volume, results remained statistically significantly lower with the Harmonic scalpel in all sensitivity analyses (Table 4). Also, when studies with a higher risk of bias were removed, it is interesting to note that the Harmonic scalpel significantly reduced the risk of postoperative complications compared to conventional techniques $(P=0.03)$. For hospital length of stay and transfusion risk, there were an inadequate number of studies to conduct a full meta-analysis for some sensitivity variables. Additionally, results of the primary analysis were insensitive to whether or not conventional methods focused solely on monopolar electrosurgery or when the imputed results by Wilhelm et al. [23], Lu et al. [24], and Inoue et al. [26] were excluded for operating time, drainage volume, or all continuous variables, respectively.

\section{Discussion}

Given the challenges associated with conventional hemostatic techniques in gastric surgery, advanced devices that can overcome these drawbacks and improve surgical outcomes may be preferred. This meta-analysis assessed the performance of the Harmonic scalpel compared to electrosurgery or suture ligation in gastrectomy. The breadth of the systematic search was quite extensive, with a large quantity of international, national, and regional databases searched.

The findings of the meta-analysis support the benefit of using the Harmonic scalpel in surgery, with a significant reduction in operating time, drainage volume, and intraoperative blood loss, in comparison to conventional methods. Although no significant differences between groups were 


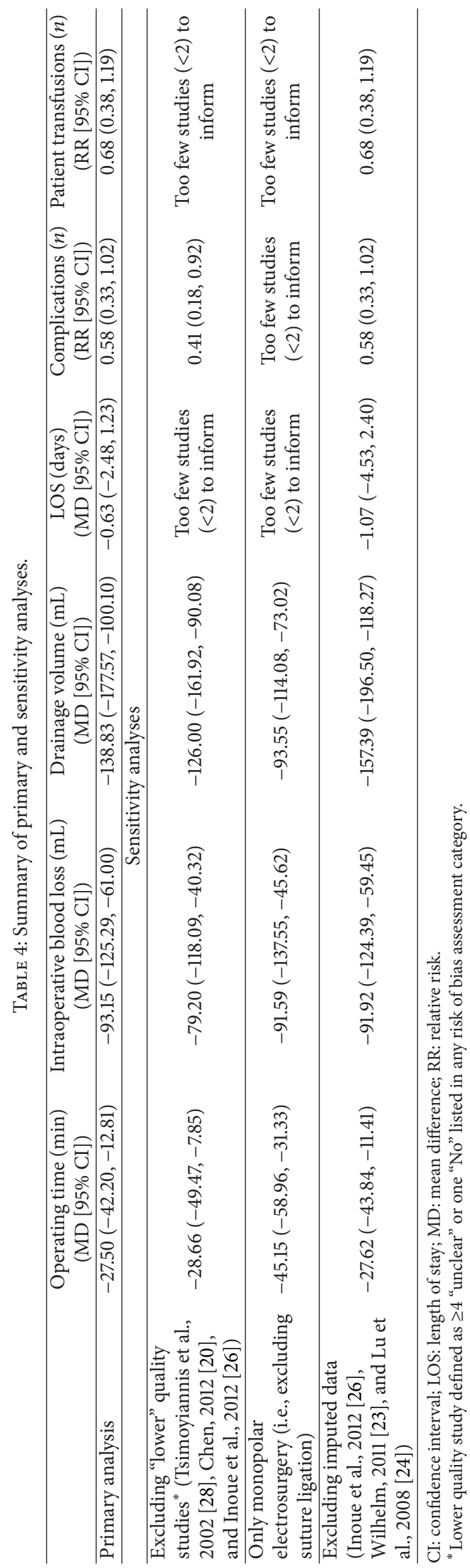




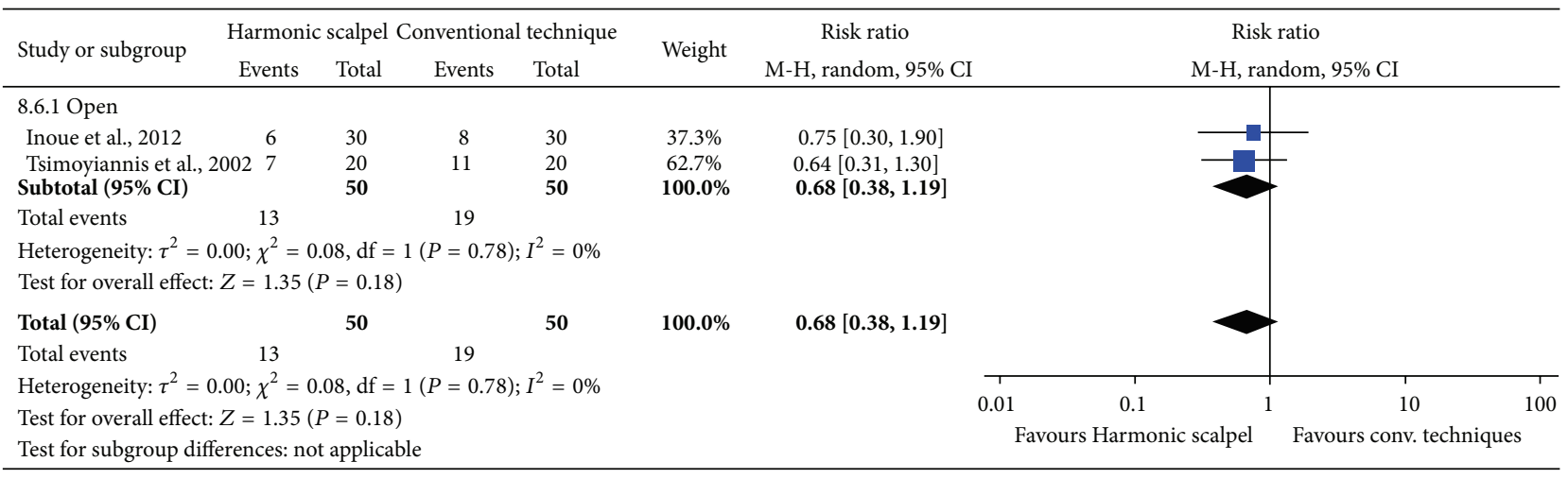

FIGURE 7: Forest plot of meta-analysis results for patient transfusion risk.

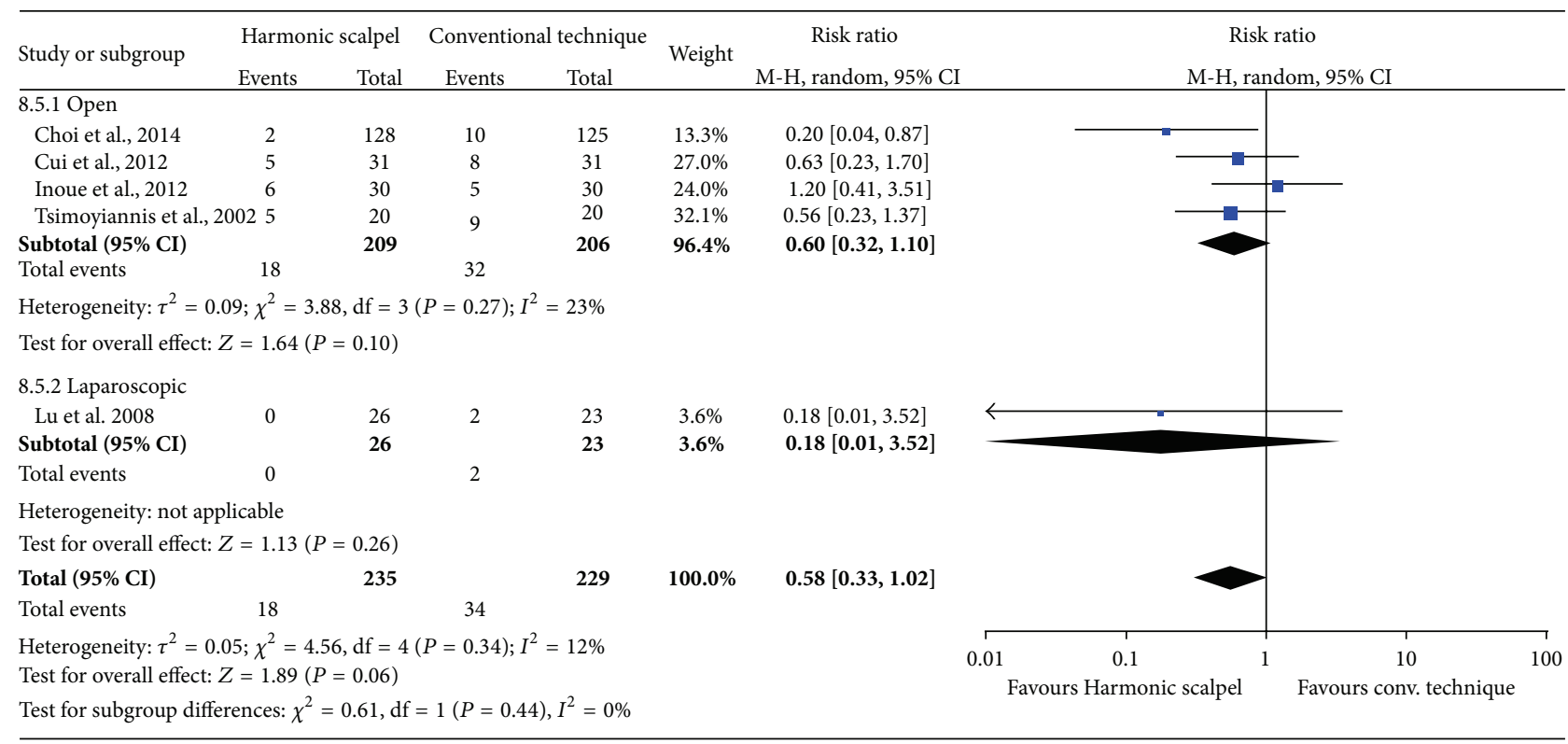

FIGURE 8: Forest plot of meta-analysis results for total complication rate.

observed for other outcome measures, results numerically favored the Harmonic scalpel for reducing length of hospital stay, transfusions, and postoperative complications. The main reported complications for both study groups included postoperative bleeding, chylous leakage, gastrointestinal paralysis, and wound infection. Results for all outcome measures remained relatively consistent when subgroup and sensitivity analyses were conducted, which restricted included studies by type of conventional device, type of surgery, and data imputation of results, highlighting the robustness of the findings. However, sensitivity analyses excluding lower quality studies produced a significant reduction in the risk of complications with the Harmonic scalpel. Methods outlined by Cochrane [25] were used to evaluate the quality and potential bias of the included studies. Results showed that the quality of included studies was acceptable overall, with fairly low risk of bias.

A more general meta-analysis by Chen et al. [22] compared the use of ultrasonic scalpels to conventional techniques in gastrectomy, providing evidence that these devices can reduce operating time and intraoperative blood loss, without compromising patient safety. Chen et al. [22] conducted analyses using both randomized and observational studies. The results of our meta-analysis confirm the findings of this published study, add more recent data, include only RCTs, and exclusively assess Harmonic scalpel use in gastrectomy. Our findings are also aligned with several meta-analyses that evaluated the efficacy and safety of this ultrasonic device in patients undergoing thyroid surgery $[10$, 12]. In the recent analysis of thyroidectomy by Contin et al. [12], the Harmonic scalpel significantly reduced operating time, blood loss, and postoperative drainage compared to other hemostatic techniques, demonstrating the clear benefits across surgical areas [10]. Furthermore, evidence demonstrates that the favourable outcomes associated with the Harmonic scalpel can translate into cost-savings for hospital institutions $[33,34]$.

Although all the studies included within this analysis performed gastrectomy plus D2 lymphadenectomy, it is 
important to note that the extent of lymphadenectomy performed alongside gastric resection differs between countries and institutions [7]. While some suggest that a less extensive D1 lymphadenectomy is the minimum standard for gastric cancer, this is not universally implemented in lower incidence countries (e.g., United States). Conversely, in Eastern Asia, where nearly half of gastric cancer cases occur, D2 lymphadenectomy is typically the standard procedure performed. Prospective studies in both the East and West have shown that less extensive lymphadenectomies likely result in understaging of patients and increased locoregional recurrence; yet the effect on overall survival is more challenging to determine. Further randomized studies in Western patients are required in order to make conclusions regarding the survival benefits of D2 lymphadenectomy.

This study does have limitations. First, not all data were available to inform variance inputs for the meta-analysis. In order to overcome this, imputation methods and assumptions outlined by Cochrane were utilized [25]. Sensitivity analyses excluding outcomes with imputed data showed that results remained similar to the primary analysis. Second, since the heterogeneity between studies was significant for the majority of the meta-analyses, a random-effects model was used to pool all outcome data. Third, data were not available from all studies for each of the included outcomes to be statistically combined in this meta-analysis, and four of the studies had less than 50 subjects. Therefore, some outcome results may not be as rigorous due to small sample sizes. Additionally, not all studies reported the rate of total postoperative complications; therefore the total rates included in the metaanalysis represent an addition of individual complications reported in the studies. Last, because the authors did not have access to the study protocols of the included publications, there is potential for some variation in the study quality. The exclusion of lower quality studies in sensitivity analyses produced a significant reduction in the risk of postoperative complications; however, this exclusion did not impact all other outcomes. Furthermore, this study should be viewed as representative of outcomes for open gastrectomy, since only one of the ten studies was performed laparoscopically. The single laparoscopic study did not evaluate operative time, blood loss, hospital stay, or patient transfusion risk. Exclusion of the laparoscopic study did not have a substantial impact on the parameters that were measured therein, namely, drainage volume and total complication rate.

In summary, the findings of this study hold practical importance. Reductions in blood loss may translate into a lower clinical burden for patients. Relevant to physicians and economic stakeholders, reductions in resource use have potential cost implications for hospitals. For example, reductions in intraoperative blood loss can decrease the need for blood loss management resources, such as expensive blood products, and hemostatic agents. Further, several studies have revealed the high costs associated with operating time [3537]; hence the use of time-saving devices in surgery can lead to substantial savings. Essentially, products that require fewer people and less steps are more time-cost efficient. These savings in operating time and other resources (e.g., reduced hospital stay) can help to offset the product acquisition cost.
However, there is still a need for further costing studies to fully elucidate this impact of resource aversion on potential cost savings.

\section{Conclusions}

The Harmonic scalpel is an effective surgical technique compared to conventional methods in gastrectomy and lymphadenectomy. It offers several clinical advantages, including reduced operating time and blood loss, which can ultimately benefit the surgeon, patient, and hospital, without the addition of safety concerns.

\section{Conflict of Interests}

Hang Cheng, Chia-Wen Hsiao, Jeffrey W. Clymer, Michael L. Schwiers, and Edward Chekan are employees of Ethicon, Inc., manufacturer of the Harmonic Scalpel. Bryanna N. Tibensky, Leena Patel, and Nicole C. Ferko are employees of Cornerstone Research Group, who were sponsored to perform this study by Ethicon, Inc.

\section{Acknowledgment}

This work was supported by Ethicon, Inc., manufacturer of the Harmonic scalpel, that provided funding to conduct the analysis and prepare the paper.

\section{References}

[1] M. den Dulk, M. Verheij, A. Cats, E. P. M. Jansen, H. H. Hartgrink, and C. J. H. van de Velde, "The essentials of locoregional control in the treatment of gastric cancer," Scandinavian Journal of Surgery, vol. 95, no. 4, pp. 236-242, 2006.

[2] A. Jemal, F. Bray, M. M. Center, J. Ferlay, E. Ward, and D. Forman, "Global cancer statistics," CA Cancer Journal for Clinicians, vol. 61, no. 2, pp. 69-90, 2011.

[3] E. van Cutsem, C. van de Velde, A. Roth et al., "Expert opinion on management of gastric and gastro-oesophageal junction adenocarcinoma on behalf of the European Organisation for Research and Treatment of Cancer (EORTC) - gastrointestinal cancer group," European Journal of Cancer, vol. 44, no. 2, pp. 182-194, 2008.

[4] R. Ladeiras-Lopes, A. K. Pereira, A. Nogueira et al., "Smoking and gastric cancer: systematic review and meta-analysis of cohort studies," Cancer Causes \& Control, vol. 19, no. 7, pp. 689701, 2008.

[5] L. Fuccio, R. M. Zagari, L. H. Eusebi et al., "Meta-analysis: can Helicobacter pylori eradication treatment reduce the risk for gastric cancer?" Annals of Internal Medicine, vol. 151, no. 2, pp. 121-128, 2009.

[6] J. A. Ajani, D. J. Bentrem, S. Besh et al., "Gastric cancer, version 2.2013: featured updates to the NCCN Guidelines," Journal of the National Comprehensive Cancer Network, vol. 11, no. 5, pp. 531-546, 2013.

[7] B. Schmidt and S. S. Yoon, "D1 versus D2 lymphadenectomy for gastric cancer," Journal of Surgical Oncology, vol. 107, no. 3, pp. 259-264, 2013.

[8] A. Manouras, H. Markogiannakis, A. S. Koutras et al., "Thyroid surgery: comparison between the electrothermal bipolar vessel 
sealing system, harmonic scalpel, and classic suture ligation," American Journal of Surgery, vol. 195, no. 1, pp. 48-52, 2008.

[9] G. Bandi, C. C. Wen, E. A. Wilkinson, S. P. Hedican, T. D. Moon, and S. Y. Nakada, "Comparison of blade temperature dynamics after activation of Harmonic Ace Scalpel and the Ultracision Harmonic Scalpel LCS-K5," Journal of Endourology, vol. 22, no. 2, pp. 333-336, 2008.

[10] T. Ecker, A. L. Carvalho, J.-H. Choe, G. Walosek, and K. J. Preuss, "Hemostasis in thyroid surgery: harmonic scalpel versus other techniques-a meta-analysis," Otolaryngology-Head and Neck Surgery, vol. 143, no. 1, pp. 17-25, 2010.

[11] F. Sista, M. Schietroma, C. Ruscitti et al., "New ultrasonic dissector versus conventional hemostasis in thyroid surgery: a randomized prospective study," Journal of Laparoendoscopic \& Advanced Surgical Techniques, vol. 22, no. 3, pp. 220-224, 2012.

[12] P. Contin, K. Gooßen, K. Grummich et al., "ENERgized vessel sealing systems versus CONventional hemostasis techniques in thyroid surgery-the ENERCON systematic review and network meta-analysis," Langenbeck's Archives of Surgery, vol. 398, no. 8, pp. 1039-1056, 2013.

[13] G. Materazzi, G. Caravaglios, V. Matteucci, A. Aghababyan, M. Miccoli, and P. Miccoli, "The impact of the Harmonic FOCUS on complications in thyroid surgery: a prospective multicenter study," Updates in Surgery, vol. 65, no. 4, pp. 295-299, 2013.

[14] V. Mahabaleshwar, L. Kaman, J. Iqbal, and R. Singh, "Monopolar electrocautery versus ultrasonic dissection of the gallbladder from the gallbladder bed in laparoscopic cholecystectomy: a randomized controlled trial," Canadian Journal of Surgery, vol. 55, no. 5, pp. 307-311, 2012.

[15] S. A. Ramzanali, Zia-ul-Islam, and S. S. Shah, "Monopolar electrocautery versus ultrasonic dissection of the gallbladder from the gallbladder bed in laparoscopic cholecystectomy," Journal of Ayub Medical College, vol. 25, no. 3-4, pp. 16-18, 2013.

[16] M. Marino, R. Rimonda, M. E. Allaix, G. Giraudo, and C. Garrone, "Ultrasonic versus standard electric dissection in laparoscopic colorectal surgery: a prospective randomized clinical trial," Annals of Surgery, vol. 242, no. 6, pp. 897-901, 2005.

[17] R. Campagnacci, A. de Sanctis, M. Baldarelli, M. Rimini, G. Lezoche, and M. Guerrieri, "Electrothermal bipolar vessel sealing device vs. ultrasonic coagulating shears in laparoscopic colectomies: a comparative study," Surgical Endoscopy and Other Interventional Techniques, vol. 21, no. 9, pp. 1526-1531, 2007.

[18] M. Hubner, N. Demartines, S. Muller, D. Dindo, P.-A. Clavien, and D. Hahnloser, "Prospective randomized study of monopolar scissors, bipolar vessel sealer and ultrasonic shears in laparoscopic colorectal surgery," The British Journal of Surgery, vol. 95, no. 9, pp. 1098-1104, 2008.

[19] B. Matthews, L. Nalysnyk, R. Estok et al., "Ultrasonic and nonultrasonic instrumentation: a systematic review and metaanalysis," Archives of Surgery, vol. 143, no. 6, pp. 592-600, 2008.

[20] C. Chen, "The application of ultrasonically activated scalpel in radical resection of gastric carcinoma," Guide of China Medicine, vol. 10, no. 8, pp. 345-347, 2012.

[21] M.-G. Choi, S. J. Oh, J. H. Noh, T. S. Sohn, S. Kim, and J. M. Bae, "Ultrasonically activated shears versus electrocautery in open gastrectomy for gastric cancer: a randomized controlled trial," Gastric Cancer, vol. 17, no. 3, pp. 556-561, 2014.

[22] X. Chen, Z. Lu, L. Wang et al., "Comparison of ultrasonic scalpel versus conventional techniques in open gastrectomy for gastric carcinoma patients: a systematic review and meta-analysis," PLoS ONE, vol. 9, no. 7, Article ID e103330, 2014.
[23] D. Wilhelm, M. Szabo, F. Glass, C. Schuhmacher, H. Friess, and H. Feussner, "Randomized controlled trial of ultrasonic dissection versus standard surgical technique in open left hemicolectomy or total gastrectomy," The British Journal of Surgery, vol. 98, no. 2, pp. 220-227, 2011.

[24] W. Lu, G. Zhao, and X. Qin, "Usage of new type ultrasonically activated scalpel in radical resection of gastric carcinoma," Chinese Journal of Practical Surgery, vol. 28, no. 3, pp. 203-205, 2008.

[25] J. Higgins and S. Green, The Cochrane Handbook for Systematic Reviews of Interventions, 2011, http://www.cochrane-handbook .org/.

[26] K. Inoue, Y. Nakane, T. Michiura et al., "Ultrasonic scalpel for gastric cancer surgery: a prospective randomized study," Journal of Gastrointestinal Surgery, vol. 16, no. 10, pp. 1840-1846, 2012.

[27] S. P. Hozo, B. Djulbegovic, and I. Hozo, "Estimating the mean and variance from the median, range, and the size of a sample," BMC Medical Research Methodology, vol. 5, article 13, 2005.

[28] E. C. Tsimoyiannis, M. Jabarin, J. C. Tsimoyiannis, J. P. Betzios, C. Tsilikatis, and G. Glantzounis, "Ultrasonically activated shears in extended lymphadenectomy for gastric cancer," World Journal of Surgery, vol. 26, no. 2, pp. 158-161, 2002.

[29] L. Liu, Z. Zhou, and J. Lu, "Application of ultracision harmonic scalpel in radical resection of gastric carcinoma," China Oncology, vol. 1, no. 20, pp. 799-801, 2010.

[30] Y. Wang, J. Gu, R. Guo, J. Zhang, and C. Gu, "Clinical value and skills of application of ultracision scapel in radical resection of gastric cancer," Chinese Journal of Clinical Oncology, vol. 37, no. 23, pp. 1370-1372, 2010.

[31] W. Cui, F. Zuo, G. Chen, S. Li, and B. Yu, "Analysis on the application of ultrasonically activated scalpeI in radical surgery for proximal gastric cancer," Clinical Journal of Medical Officers, vol. 40, no. 6, pp. 1299-1302, 2012.

[32] P. Xu, F. Wang, Y. Luan, and Z. Pan, "Observation of clinical effect of ultracision-harmonic scalpel assisted radical resection of gastric cancer," People’s Military Surgeon, vol. 53, no. 7, pp. 490-491, 2010.

[33] J. Ortega, C. Sala, B. Flor, and S. Lledo, "Efficacy and costeffectiveness of the UltraCision harmonic scalpel in thyroid surgery: an analysis of 200 cases in a randomized trial," Journal of Laparoendoscopic \& Advanced Surgical Techniques Part A, vol. 14, no. 1, pp. 9-12, 2004.

[34] M. Ruggeri, R. Dibidino, M. Marchetti, C. P. Lombardi, M. Raffaelli, and A. Cicchetti, "The harmonic study: cost-effectiveness evaluation of the use of the ultrasonic scalpel in total thyroidectomy," International Journal of Technology Assessment in Health Care, vol. 28, no. 3, pp. 259-264, 2012.

[35] F. Kallinowski, T. Pfeil, and W. Ulbrich, "Quality management in surgical intervention-a prospective outcomes research study of fleece-bound tissue gluing," Gesundheitsokonomie und Qualitatsmanagement, vol. 10, no. 3, pp. 151-160, 2005.

[36] R. Shippert, "A study of time-dependent operating room fees and how to save $\$ 100000$ by using time-saaving products," American Journal of Cosmetic Surgery, vol. 22, no. 1, pp. 25-34, 2005.

[37] K. W. Park and C. Dickerson, "Can efficient supply management in the operating room save millions?" Current Opinion in Anaesthesiology, vol. 22, no. 2, pp. 242-248, 2009. 


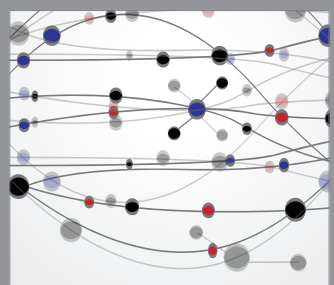

The Scientific World Journal
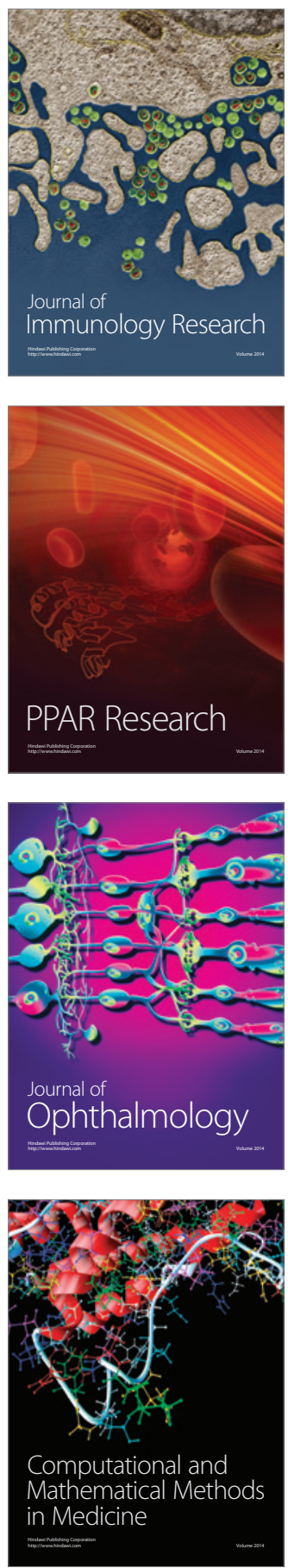

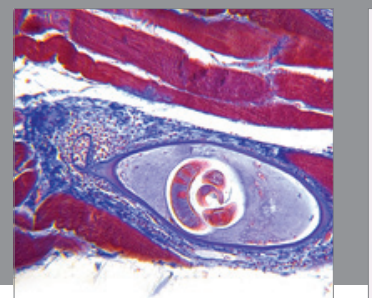

Gastroenterology

Research and Practice
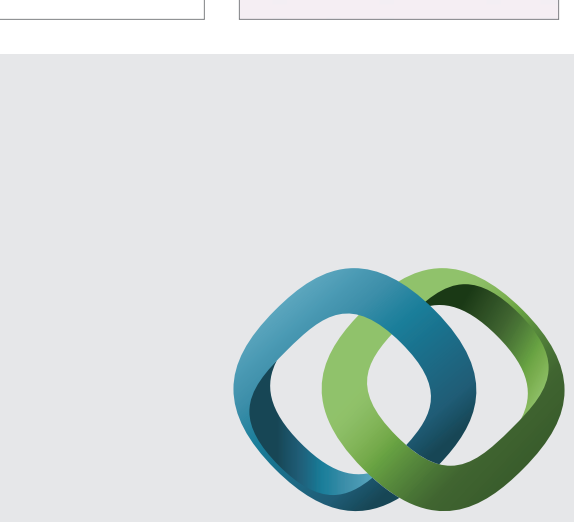

\section{Hindawi}

Submit your manuscripts at

http://www.hindawi.com
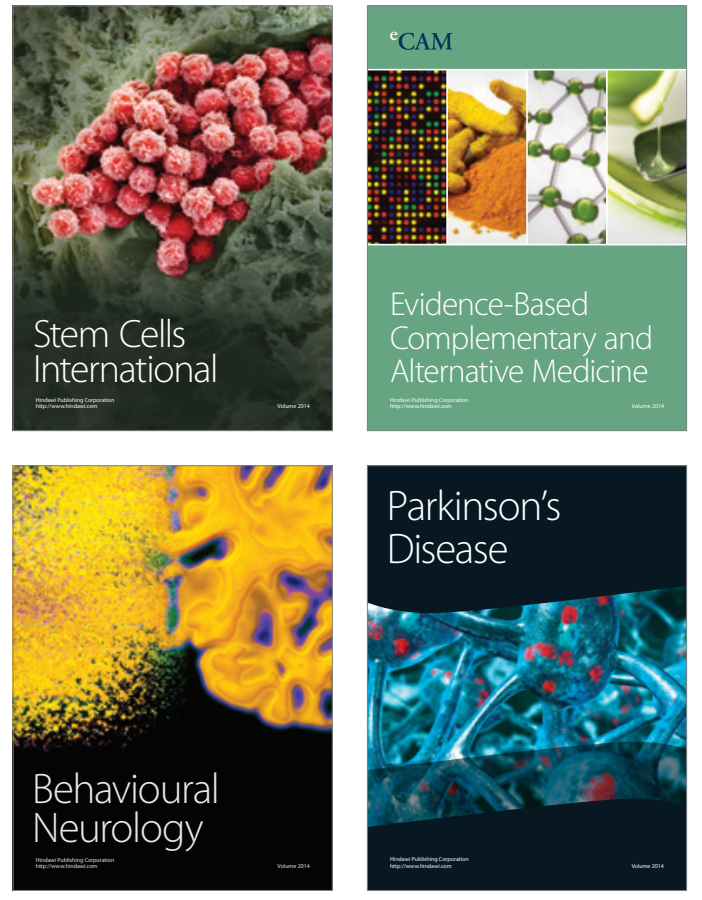
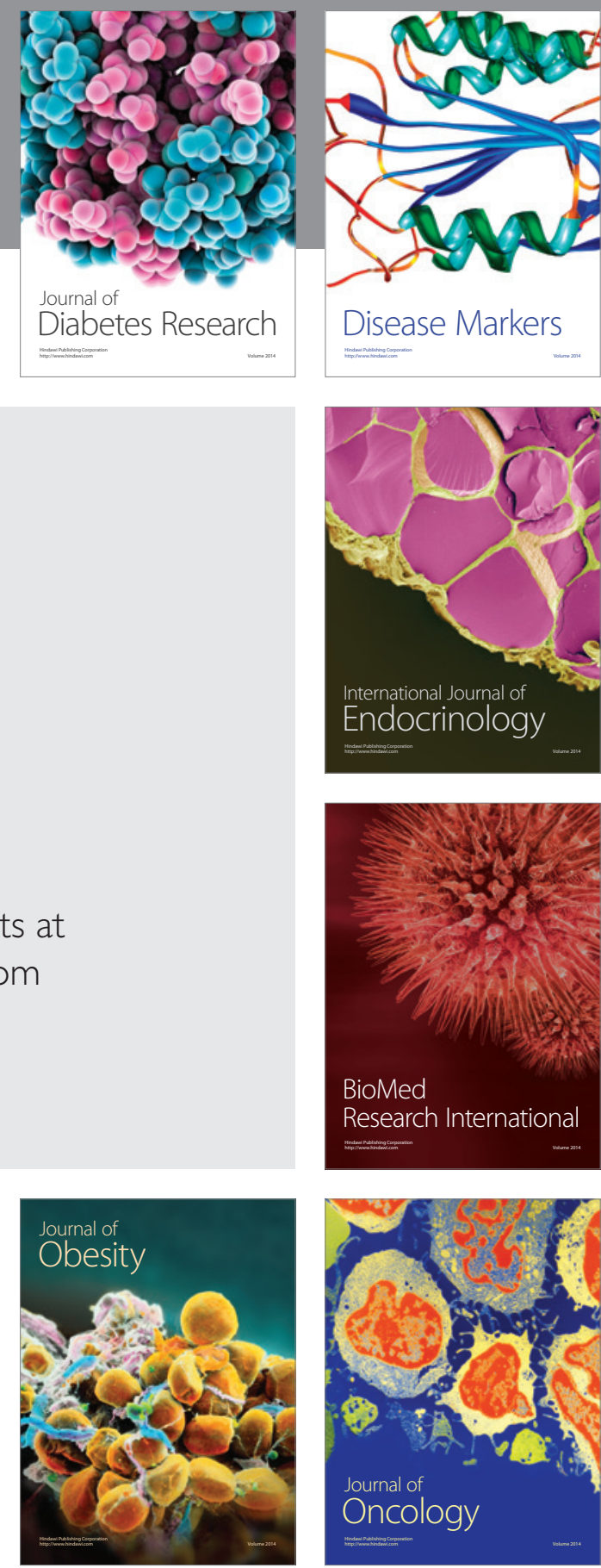

Disease Markers
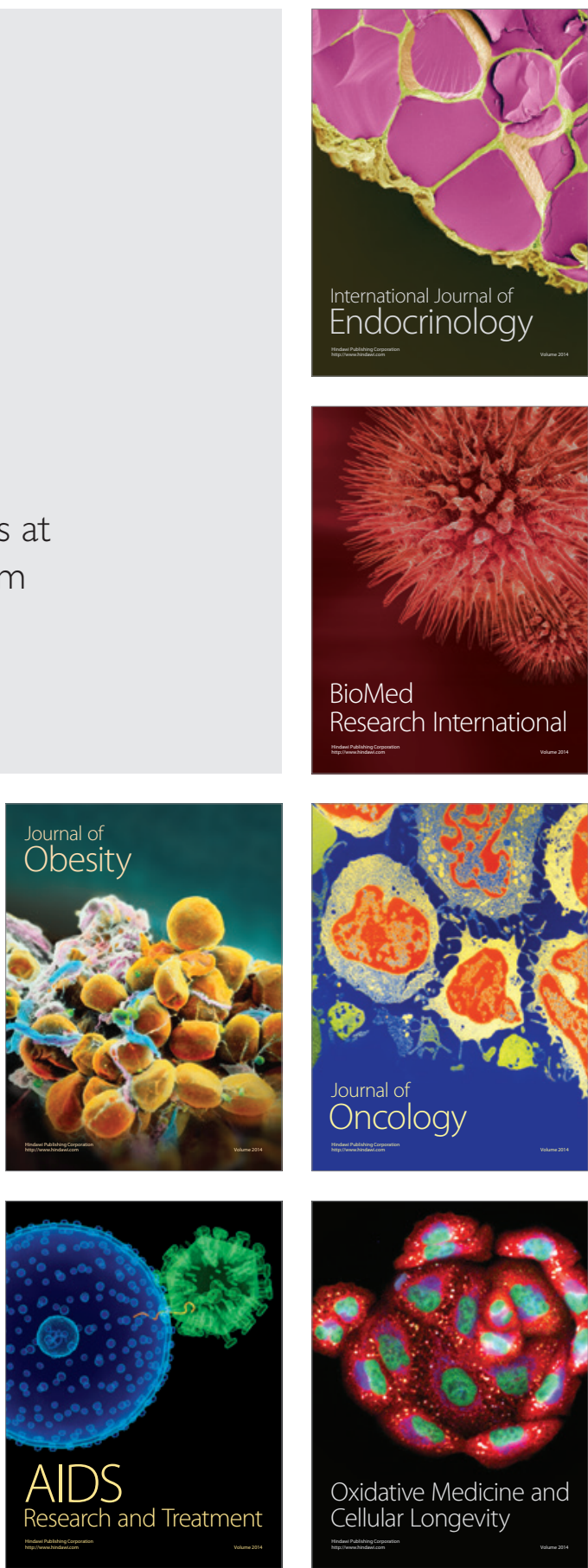